tivity which may amount to disruption. It is difficult, however, to see how the energy to feed the disruption, or the spin-up of a galaxy, can be produced quickly enough in normal galaxies, with their observed central densities. A hypothesis of Layzer that galaxies form by agglomeration of matter, and not by fragmentation, falls down, as Gribbin notes, because the material which makes planets seem observationally to have come out of stars, whereas during agglomeration planets would form first.

Having thus surveyed earlier work, the author turns to what he sees as a synthesis combining some attractive features of the continuous creation cosmology and currently accepted views of a singular explosive origin for the universe. Essentially, galaxies are little pockets of the universe having galactic mass whose cosmogonic expansion has been somehow delayed.
Since each pocket starts close to its Schwarzschild radius, there is enough free energy available to bring about high energy outbursts typical of quasars. A quiet galaxy is formed later in the evolutionary sequence, either as an elliptical, when little material surrounds the initial pocket, or a spiral, if it is surrounded by gas and dust. As a picture this raises as many problems as it solves. Above all, the basis for postulation is entirely arbitrary on the present state of the evidence, at least as arbitrary as alternative hypotheses in predicting galaxies with an observed mass distribution. This is treated very lightly as a 'boundary value problem' dependent on the initial conditions of the universe at the time of the big bang, but this approach is without much predictive power, and the weaker for that.

On the cover John Gribbin's book is described as "suitable for the second year student of physics" as indeed it is in some ways. Most physicists, as opposed to astrophysicists, will find the abundance of hand-waving arguments and the tendency to cull formalism from the air thoroughly infuriating, but in a topic as variegated and alive as this, it is one of the effective ways of introducing ideas seriously, without the dead weight of rigour. The author is aware of pitfalls, and not to be strongly faulted here. As a useful summary of a tangled topic this volume should find its place on many a professional's bookshelf, and on those of students who can afford it.

John E. Beckman

John Beckman is a Senior Research Astronomer in the Astronomy Division of the European Space Research Centre, Noordwijk, Netherlands.

\section{Mössbauer again}

Principles of Mössbauer Spectroscopy. (Studies in Chemical Physics.) By T. C. Gibb. Pp. 254. (Chapman and Hall: London, Halsted: New York, March 1976.) £9.

YET another book on the principles of Mössbauer spectroscopy! The author in fact lists seven similar texts in his bibliography although admittedly only three of these cover the same field of chemical applications.

The basic format consists of two chapters concerned with the general principles of the technique followed by nine chapters in which the applications are illustrated with some 200 references to published work. The main body of the book is made up of three chapters in which the use of the measured hyperfine interactions between the nucleus and its electronic surroundings is discussed, both in the study of chemical bonding, and also in structure determinations of molecular complexes. Here, as elsewhere, the examples are not restricted to the well studied isotope ${ }^{57} \mathrm{Fe}$ alone, but cover the use of tin, antimony, iodine, ruthenium and iridium isotopes as well as several lanthanides and actinides. These chapters are followed by one in which the effects on the Mössbauer resonance of the dynamic processes involving lattice vibrations, atomic diffusion and spin relaxation are discussed; and one concerned with the structures and magnetic properties of spinels. The use of Mössbauer isotopes as impurity probes is illustrated including a mention of the novel experiments to measure the hyperfine interactions at an isolated Mössbauer atom obtained by condensation with an inert gas. Finally there are chapters outlining studies of the effects of atomic environment in alloys, analytical applications and the properties of iron atoms in protein molecules.

Throughout the style is clear and readable. No attempt is made to discuss the instrumentation required and the use of mathematics is kept to a minimum; but there is nevertheless a lot of information packed in some 200

\section{Pharmacological marriage}

Behavioural Pharmacology. By Susan D. Iversen and Leslie L. Iversen. Pp. xiv +310. (Oxford University: New York and London, 1975.) £2.75 paper; $£ 5.50$ boards.

THE Iversens' marriage of neuro- and psychopharmacology has produced a remarkably sophisticated, yet readable, text on behavioural pharmacology. The text is self-contained and appropriate for advanced undegraduate or firstyear graduate students. A wide spectrum of drugs are included: amphetamines, barbiturates, hormones, anti-depressants, opiates, benzodiazepines, phenothiazines and butyrophenones, hallucinogens and cannabis.

The neurochemical chapters cover broadly but carefully the basic enzymology and pharmacology of all the neurotransmitters. Modern techniques, such as histofluorescence and iontophoresis, are introduced; and methodological problems are discussed, such as avoiding the blood-brain barrier in pharmacological experiments.

The behavioural chapters are written search in psychopharmacology at from the perspective of 'descriptive Massachusetts General Hospital, behaviourism', with emphasis on Boston, Massachusetts. pages. There are similar texts available but this present volume probably covers a wider range of measurements and should prove a useful introduction for the student. G. Longworth

Dr Longworth is a Senior Scientific Officer in the Nuclear Physics Division of the Atomic Energy Research Establishment, Harwell, UK.

schedules of reinforcement. Coverage is sufficiently critical and thorough to be useful for research workers as well as students. Distinctive profiles emerge for drugs such as benzodiazepines (release of behaviour suppressed by punishment) and phenothiazines (decrease of discriminative stimulus control). Ethologically-minded readers may be distressed to see changes in rate of responding after increases or decreases in the size of reinforcement offered as a model for elation and deduced in primates by separation are dismissed as "a difficult condition to induce experimentally". There are reliable, quantitative paradigms, on which schizophrenic patients are known to differ from normals. One of them, the 'continuous performance test' of Kornetsky, has an animal version, but others-such as the series of reaction time paradigms developed by Shakow and his school-have not been reproduced in animals. One might hope that a literature of this kind would develop sufficiently to be included in a second edition of this excellent text.

Steven Matthysse is carrying out re- Boston, Massachusetts. pression, whereas depressive states in-

Steven Matthysse 